\title{
Facile synthesis of 12-carboxamido-11-spirostenes via palladium-catalyzed carbonylation reactions
}

\author{
Péter Ács ${ }^{a}$, Ernö Müller ${ }^{a}$, Gábor Czira ${ }^{b}$, Sándor Mahó ${ }^{b}$, \\ Mariette Perreirac ${ }^{c}$, László Kollár ${ }^{a, d, *}$ \\ a University of Pécs, Department of Inorganic Chemistry, Ifjúság u.6., P.O. Box 266, H-7624 Pécs, Hungary \\ ${ }^{\mathrm{b}}$ Gedeon Richter Pharmaceutical Work Ltd., Budapest, Hungary \\ c University of Coimbra, Department of Chemistry, Rua Larga, 3049 Coimbra, Portugal \\ d Research Group for Chemical Sensors of the Hungarian Academy of Sciences, P.O. Box 266, H-7624 Pécs, Hungary
}

\section{A R T I C L E I N F O}

\section{Article history:}

Received 7 April 2006

Received in revised form 29 May

2006

Accepted 31 May 2006

Published on line 7 July 2006

\section{Keywords:}

Iodo-alkenene

Carboxamide

Hecogenin

Palladium-catalyst

Carbonylation

\begin{abstract}
A B S T R A C T
12-Carboxamido- and 12-carboxyl-11-spirostenes were synthesized from the corresponding 12-iodo-11-ene derivative in palladium-catalyzed carbonylation reactions under mild reaction conditions. The synthesis of the iodo-alkene substrate is based on the transformation of the 12-keto derivative (hecogenin) to hydrazone, which was treated with iodine in the presence of a base (1,1,3,3-tetramethyl guanidine). While various 12-carboxamides were synthesized in moderate to high yields by using simple alkyl/arylamines or amino acid methylesters as $\mathrm{N}$-nucleophiles, low yields can be achieved with alcohols as O-nucleophiles. The homogeneous carbonylation reactions tolerate the 3-hydroxy substituent and the spiroacetal moiety.
\end{abstract}

@ 2006 Elsevier Inc. All rights reserved.

\section{Introduction}

The homogeneous catalytic functionalization of various skeletons, among them biologically important ones, is an efficient method for the synthesis of new derivatives [1-3]. There is an increasing interest in developing new strategies to introduce functional groups into specific positions of the steroidal nuclei in order to modify their biological properties. Transition metal catalyzed reactions are versatile tools both for the construction of the steroid framework from easily available building blocks and for the functionalization of the steroidal skeleton [4].

The ester and carboxamide functionalities (especially at the distinguished position-17 or 3 of an estrene [5-8] or androstane skeleton [9-11]) proved to be efficient moieties in pharmacologically important derivatives and can be obtained in palladium-catalyzed reactions. Although the functionalization of the A and D ring of the steroidal skeleton is straightforward also in other homogeneous catalytic reactions (crosscoupling, dihydroxylation, hydroformylation, etc.) [4], to the best of our knowledge, no examples for the carbonylation reactions or any other carbon-carbon bond forming reactions at the sterically hindered positions (C-11 and C-12) of the C-ring are known.

In the present paper, we report on the efficient synthesis of steroids possessing 12-carboxamido-11-ene moiety in palladium-catalyzed carbonylation of an 'iodo-vinyl' substrate bearing 12-iodo-11-ene functionality. The application of the

\footnotetext{
* Corresponding author. Tel.: +36 72 503600; fax: +36 72501527 .

E-mail address: kollar@ttk.pte.hu (L. Kollár).
} 
easily accessible iodo-alkenes [12,13] as substrates provides an approach for the synthesis of 12-functionalized steroids of potential practical importance.

\section{Experimental}

$\mathrm{PPh}_{3}$ and 1,1,3,3-tetramethylguanidine (TMG) were purchased from Aldrich. Hecogenin was obtained from the Gedeon Richter Pharmaceutical Work Ltd., Commercial $\mathrm{Et}_{3} \mathrm{~N}$, primary and secondary amines (Aldrich) were used without further purification. Toluene, DMF, and the alcohols were dried according to standard procedures.

The steroidal 12-iodo-11-ene (3) was synthesized according to an analogous method [13] by using the 12-keto derivative (1). It was converted into the corresponding hydrazone (2) that was treated with iodine in the presence of TMG resulting in 3. Owing to differences to the previously published methods, a detailed description of the synthesis will be given below.

The ${ }^{1} \mathrm{H}$ and ${ }^{13} \mathrm{C}$ NMR spectra were recorded on a VARIAN INOVA 400 spectrometer at 400 and $100.58 \mathrm{MHz}$, respectively. The chemical shifts are given as $\delta$ values (ppm), with tetramethylsilane as the internal standard. TLC analyses were carried out by using Merck TLC sheets (Silica gel $60 \mathrm{~F}_{254}$ ) and chloroform as well as chloroform/ethanol (19/1) as eluents.

Mass spectra were recorded on a Finnigan MAT 95 magnetic sector instrument equipped with a FAB ion source. The $\mathrm{Cs}^{+}$ion gun was used at $20 \mathrm{keV}$ and the matrix was glycerol.

\subsection{Synthesis of $3 \beta$-hydroxy-12-iodo- $5 \alpha, 25 R-$ spirost-11-ene (3)}

Hecogenin (1) (4 g, $9.29 \mathrm{mmol})$, freshly distilled hydrazine hydrate $(98 \%, 40.24 \mathrm{~g}, 0.81 \mathrm{~mol})$ and barium oxide $(40 \mathrm{mg})$ in ethylene glycol $(150 \mathrm{ml})$ were heated for 4 days at $160^{\circ} \mathrm{C}$. After completion of the reaction the mixture was poured onto water and extracted with dichloromethane. Then the organic phase was dried over sodium sulfate, and evaporated to give hecogenin-hydrazone (2). The product was used in the next step without further purification.

To a stirred solution of iodine $(6.08 \mathrm{~g}, 23.92 \mathrm{mmol})$ in ether (80 ml) 1,1,3,3-tetramethylguanidin (TMG, $9.27 \mathrm{~g}, 80.48 \mathrm{mmol}$ ) was added slowly and cooled by iced water bath during the addition. The solution of hecogenin-hydrazone (4 $\mathrm{g}$, $8.99 \mathrm{mmol})$ in ether $(20 \mathrm{ml})$ was added dropwise at room temperature. After the addition was completed, the mixture was stirred for an hour. Then the solvent was evaporated and the residue was heated at $90^{\circ} \mathrm{C}$ under argon atmosphere for $2 \mathrm{~h}$. The mixture was poured onto water and extracted with ether. The combined organic layer was washed with $1 \mathrm{~N}$ aqueous $\mathrm{HCl}$, water, $5 \%$ aqueous $\mathrm{NaHCO}_{3}$, water, saturated aqueous $\mathrm{Na}_{2} \mathrm{~S}_{2} \mathrm{O}_{3}$ and water again, dried on sodium sulfate and evaporated. Purification by column chromatography (silicagel, benzene/ethyl acetate (7:3)) gave pure 3 as a white solid. Yield: $2.55 \mathrm{~g}(52.5 \%)$.

\subsection{General procedure for the hydroxycarbonylation reaction (synthesis of 4 )}

A mixture of $3(300 \mathrm{mg}, 0.55 \mathrm{mmol})$, palladium(II) acetate $(5.6 \mathrm{mg}, \quad 0.025 \mathrm{mmol})$, and triphenylphosphine $(13.1 \mathrm{mg}$,
$0.05 \mathrm{mmol}$ ) were dissolved in a mixture of $10 \mathrm{ml}$ aqueous DMF (containing $1 \%$ water) and triethylamine $(0.5 \mathrm{ml})$ under argon. The atmosphere was changed to carbon monoxide ( 1 bar), and the reaction was conducted at $50^{\circ} \mathrm{C}$ for $6 \mathrm{~h}$. (The composition of the reaction mixture was checked by TLC.) The solvent was evaporated to dryness, and the rest was dissolved in $10 \mathrm{ml}$ chloroform. It was washed with $15 \mathrm{ml}$ portions of water (twice), 5\% hydrochloric acid and brine. The organic layer was separated, dried on sodium sulfate and evaporated. The chromatography (silicagel, ethanol/chloroform $=1 / 19$ ) resulted in the 12-carboxyl derivative (4). Yield: $0.11 \mathrm{~g}(44 \%)$.

\subsection{General procedure for the aminocarbonylation reaction}

A mixture of $3(300 \mathrm{mg}, 0.55 \mathrm{mmol})$, palladium(II) acetate (5.6 $\mathrm{mg}, \quad 0.025 \mathrm{mmol})$, and triphenylphosphine (13.1 $\mathrm{mg}$, $0.05 \mathrm{mmol}$ ) were dissolved in $10 \mathrm{ml}$ DMF under argon. Triethylamine $(0.5 \mathrm{ml})$ and tert-butylamine $(0.293 \mathrm{ml}, 27 \mathrm{mmol})$ or an other N-nucleophile were added. (The alanine and proline methylester as $\mathrm{N}$-nucleophile were used as hydrochloride salt and were measured into the reaction vessel together with the catalyst.) The atmosphere was changed to carbon monoxide ( 1 bar), and the reaction was conducted at $50^{\circ} \mathrm{C}$ for $6 \mathrm{~h}$. The composition of the reaction mixture was checked by TLC. The solvent was evaporated to dryness, and the rest was dissolved in $10 \mathrm{ml}$ chloroform. It was washed with $15 \mathrm{ml}$ portions of water (twice), 5\% hydrochloric acid, saturated sodium hydrocarbonate and brine. The organic layer was separated, dried on sodium sulfate and evaporated. The chromatography (silicagel, ethyl acetate/chloroform =1/1) resulted in the target 12 -tert-butyl-carboxamido derivative (7) or the corresponding amide derivative.

\subsection{Analytical and spectroscopic data of compounds}

2.4.1. 3 $\beta$-Hydroxy-12-iodo-5 $\alpha, 25 R$-spirost-11-ene (3)

${ }^{1} \mathrm{H} \mathrm{NMR}\left(\mathrm{CDCl}_{3}, 400 \mathrm{MHz}\right): 6.16$ (br s, $\left.1 \mathrm{H}, 11-\mathrm{CH}\right) ; 4.45$ (dq, $2.0 \mathrm{~Hz}$, $7.5 \mathrm{~Hz}, 1 \mathrm{H}, 16-\mathrm{CH}) ; 3.58$ (m, 1H, 3-CH); 3.45 (br dd, $10.9 \mathrm{~Hz}$, $\left.1.8 \mathrm{~Hz}, 1 \mathrm{H}, 26-\mathrm{CH}_{a} \mathrm{H}_{\mathrm{b}}\right) ; 3.35$ (t, $\left.10.9 \mathrm{~Hz}, 1 \mathrm{H}, 26-\mathrm{CH}_{\mathrm{a}} \mathrm{H}_{\mathrm{b}}\right) ; 0.90-2.2$ (m, 23H, skeleton protons); 1.25 (d, $\left.6.8 \mathrm{~Hz}, 21-\mathrm{CH}_{3}\right) ; 0.96(\mathrm{~s}, 3 \mathrm{H}$, $\left.18-\mathrm{CH}_{3}\right) ; 0.79\left(\mathrm{~s}, 3 \mathrm{H}, 19-\mathrm{CH}_{3}\right) ; 0.78\left(\mathrm{~d}, 6.3 \mathrm{~Hz}, 3 \mathrm{H}, 27-\mathrm{CH}_{3}\right) ;{ }^{13} \mathrm{C}$ NMR (100.58 MHz, CDCl $)$ ): 137.6 (11-C); 111.0 (12-C); 108.7 (22C); 79.5; 71.0; 66.9; 61.2; 60.7; 54.5; 51.7; 44.3; 41.6; 37.8; 36.1; 35.9; 33.2; 31.8; 31.4; 31.2; 30.3; 30.2; 28.8; 19.0; 17.0; 15.8; 13.0; MS $(\mathrm{m} / \mathrm{z}): 541(\mathrm{M}+\mathrm{H})^{+}, 523,481,413,397$. Analysis calculated for $\mathrm{C}_{27} \mathrm{H}_{41} \mathrm{IO}_{3}(\mathrm{M}=540.53)$ : C, 60.00; H, 7.65. Found: C, 60.11; H, 7.52. $R_{\mathrm{f}}=0.53\left(\mathrm{CHCl}_{3}\right) ; R_{\mathrm{f}}=0.59\left(\mathrm{CHCl}_{3} / \mathrm{EtOH}=19 / 1\right) ;$ m.p. $=98-100^{\circ} \mathrm{C}$ (as obtained after column chromatography).

\subsubsection{3 $\beta$-Hydroxy-12-carboxyl-5 $\alpha, 25 R$-spirost-11-ene (4)}

${ }^{1} \mathrm{H} \mathrm{NMR}\left(\mathrm{CDCl}_{3}, 400 \mathrm{MHz}\right): 6.90$ (br s, $\left.1 \mathrm{H}, 11-\mathrm{CH}\right) ; 4.48$ (dq, 7.6 Hz, $2.7 \mathrm{~Hz}, 1 \mathrm{H}, 16-\mathrm{CH}) ; 3.62$ (m, 1H, 3-CH); 3.45 (br d, $10.3 \mathrm{~Hz}, 1 \mathrm{H}$, $\left.26-\mathrm{CH}_{a} \mathrm{H}_{\mathrm{b}}\right) ; 3.35\left(\mathrm{t}, 10.3 \mathrm{~Hz}, 1 \mathrm{H}, 26-\mathrm{CH}_{\mathrm{a}} \mathrm{H}_{b}\right) ; 0.90-2.2(\mathrm{~m}, 23 \mathrm{H}$, skeleton protons); 1.09 (s, 3H, 18- $\left.\mathrm{CH}_{3}\right) ; 1.00$ (d, $\left.7.3 \mathrm{~Hz}, 21-\mathrm{CH}_{3}\right)$; $0.79\left(\mathrm{~s}, 3 \mathrm{H}, 19-\mathrm{CH}_{3}\right) ; 0.78\left(\mathrm{~d}, 6.3 \mathrm{~Hz}, 3 \mathrm{H}, 27-\mathrm{CH}_{3}\right) ;{ }^{13} \mathrm{C} \mathrm{NMR}$ (100.58 MHz, $\mathrm{CDCl}_{3}$ ): 171.0 (COOH); 141.4 (11-C); 120.0 (12-C); 109.0 (22-C); 80.0; 71.3; 67.1; 60.2; 57.7; 54.4; 45.0; 43.5; 38.2; $36.5 ; 36.3 ; 33.1 ; 32.2 ; 31.4 ; 30.8 ; 30.5 ; 30.2 ; 29.9 ; 29.2 ; 29.1 ; 19.3$; 17.3; 15.0; 13.6. Analysis calculated for $\mathrm{C}_{28} \mathrm{H}_{42} \mathrm{O}_{5}(\mathrm{M}=458.64)$ : $\mathrm{C}$, 
73.33; $\mathrm{H}$, 9.23. Found: $\mathrm{C}, 73.15 ; \mathrm{H}, 9.02 . \mathrm{R}_{\mathrm{f}}=0.38\left(\mathrm{CHCl}_{3}\right) ; \mathrm{R}_{\mathrm{f}}=0.44$ $\left(\mathrm{CHCl}_{3} / \mathrm{EtOH}=19 / 1\right) ;$ m.p. $=165^{\circ} \mathrm{C}$ (as obtained after column chromatography).

\subsubsection{3 -Hydroxy-12-tert-butyl-carboxamido- $5 \alpha, 25 R$ -}

spirost-11-ene (7)

${ }^{1} \mathrm{H} \mathrm{NMR}\left(\mathrm{CDCl}_{3}, 400 \mathrm{MHz}\right): 5.78$ (br s, $1 \mathrm{H}, 11-\mathrm{CH}$ ); 5.36 (br s, $1 \mathrm{H}, \mathrm{NH}) ; 4.42(\mathrm{dq}, 7.6 \mathrm{~Hz}, 2.7 \mathrm{~Hz}, 1 \mathrm{H}, 16-\mathrm{CH}) ; 3.60(\mathrm{~m}, 1 \mathrm{H}, 3-$ $\mathrm{CH}) ; 3.45$ (br d, $\left.11.0 \mathrm{~Hz}, 1 \mathrm{H}, 26-\mathrm{CH}_{a} \mathrm{H}_{\mathrm{b}}\right) ; 3.35$ (t, $11.0 \mathrm{~Hz}, 1 \mathrm{H}$, $\left.26-\mathrm{CH}_{\mathrm{a}} \mathrm{H}_{b}\right) ; 0.90-2.2(\mathrm{~m}, 23 \mathrm{H}$, skeleton protons); 1.32 (s, $9 \mathrm{H}$, $\mathrm{tBu}) ; 1.20$ (s, 3H, 18- $\left.\mathrm{CH}_{3}\right) ; 0.98\left(\mathrm{~d}, 7.1 \mathrm{~Hz}, 21-\mathrm{CH}_{3}\right) ; 0.78(\mathrm{~s}, 3 \mathrm{H}$, $\left.19-\mathrm{CH}_{3}\right) ; 0.76\left(\mathrm{~d}, 6.3 \mathrm{~Hz}, 3 \mathrm{H}, 27-\mathrm{CH}_{3}\right) .{ }^{13} \mathrm{C} \mathrm{NMR}(100.58 \mathrm{MHz}$, $\mathrm{CDCl}_{3}$ ): 170.4 (CON); 147.9 (11-C); 127.6 (12-C); 109.2 (22-C); 80.7; 71.0; 66.8; 58.7; 56.8; 53.8; 51.0; 45.3; 44.8; 42.4; 38.0; 36.1; 36.0; $33.2 ; 31.5 ; 31.2 ; 30.8 ; 30.2 ; 30.1 ; 29.5 ; 29.0 ; 28.8 ; 20.3 ; 17.1 ; 14.2$; 13.2. MS $(\mathrm{m} / \mathrm{z}): 514(\mathrm{M}+\mathrm{H})^{+}, 496,458,441,423,370$. Analysis calculated for $\mathrm{C}_{32} \mathrm{H}_{51} \mathrm{NO}_{4}(\mathrm{M}=513.76)$ : C, 74.81; $\mathrm{H}, 10.01$; $\mathrm{N}, 2.73$. Found: C, 75.02; $\mathrm{H}, 10.23 ; \mathrm{N}, 2.88 . \mathrm{R}_{\mathrm{f}}=0.48\left(\mathrm{CHCl}_{3}\right)$; $R_{\mathrm{f}}=0.53\left(\mathrm{CHCl}_{3} / \mathrm{EtOH}=19 / 1\right) ;$ m.p. $=252-255^{\circ} \mathrm{C}$ (recrystallyzed from ethanol).

\subsubsection{3 -Hydroxy-12-phenyl-carboxamido- $5 \alpha, 25 R$ -}

spirost-11-ene (8)

${ }^{1} \mathrm{H} \mathrm{NMR}$ (CDCl, $400 \mathrm{MHz}$ ): 7.25-7.38 (m, 5H, Ph); 6.25 (br s, $1 \mathrm{H}$, $\mathrm{NH}) ; 6.03$ (br s, 1H, 11-H); 4.40 (dq, $7.6 \mathrm{~Hz}, 2.7 \mathrm{~Hz}, 1 \mathrm{H}, 16-\mathrm{CH})$; 3.60 (m, 1H, 3-CH); 3.42 (br d, $11.2 \mathrm{~Hz}, 1 \mathrm{H}, 26-\mathrm{CH}_{\mathrm{a}} \mathrm{H}_{\mathrm{b}}$ ); 3.37 (t, $\left.11.0 \mathrm{~Hz}, 1 \mathrm{H}, 26-\mathrm{CH}_{\mathrm{a}} \mathrm{H}_{\mathrm{b}}\right) ; 0.90-2.2$ (m, 23H, skeleton protons); 1.00 (s, 3H, 18- $\left.\mathrm{CH}_{3}\right) ; 0.96\left(\mathrm{~d}, 7.3 \mathrm{~Hz}, 21-\mathrm{CH}_{3}\right) ; 0.80$ (s, 3H, 19- $\mathrm{CH}_{3}$ ); 0.76 (d, $\left.6.3 \mathrm{~Hz}, 3 \mathrm{H}, 27-\mathrm{CH}_{3}\right) .{ }^{13} \mathrm{C} \mathrm{NMR}\left(100.58 \mathrm{MHz}, \mathrm{CDCl}_{3}\right): 170.6$ (COOH); 139.2 (11-C); 129.0; 128.8; 122.9; 120.1 (12-C); 119.9; 109.2 (22-C); 80.8; 71.1; 71.0; 66.8; 62.2; 56.3; 54.3; 44.8; 41.7; 40.0; 37.7; 37.5; 37.0; 35.6; 35.2; 35.0; 32.6; 31.8; 31.4; 30.2; 28.8; 17.1; 14.4; 13.9; MS $(\mathrm{m} / \mathrm{z})$ : $534(\mathrm{M}+\mathrm{H})^{+}, 516,441$, 423. Analysis calculated for $\mathrm{C}_{34} \mathrm{H}_{47} \mathrm{NO}_{4}(\mathrm{M}=533.75)$ : C, 76.51; $\mathrm{H}, 8.88 ; \mathrm{N}$, 2.62. Found: $\mathrm{C}, 76.30 ; \mathrm{H}, 8.94 ; \mathrm{N}, 2.50 . \mathrm{R}_{\mathrm{f}}=0.49\left(\mathrm{CHCl}_{3}\right) ; R_{\mathrm{f}}=0.56$ $\left(\mathrm{CHCl}_{3} / \mathrm{EtOH}=19 / 1\right) ;$ m.p. $=260-262^{\circ} \mathrm{C}$ (as obtained after column chromatography).

\subsection{5. $3 \beta$-Hydroxy-12-(N,N-diethyl-carboxamido)-5 $\alpha, 25 R$ -} spirost-11-ene (9)

$\left.{ }^{1} \mathrm{H} \mathrm{NMR} \mathrm{(CDCl}{ }_{3}, 400 \mathrm{MHz}\right): 5.48$ (br s, $\left.1 \mathrm{H}, 11-\mathrm{CH}\right) ; 4.42$ (dq, $7.0 \mathrm{~Hz}, 2.1 \mathrm{~Hz}, 1 \mathrm{H}, 16-\mathrm{CH}) ; 3.60(\mathrm{~m}, 1 \mathrm{H}, 3-\mathrm{CH}) ; 3.20-3.45(\mathrm{~m}$, $\left.6 \mathrm{H}, 26-\mathrm{CH}_{2}+\mathrm{N}\left(\mathrm{CH}_{2} \mathrm{CH}_{3}\right)_{2}\right) ; 0.90-2.2(\mathrm{~m}, 29 \mathrm{H}$, skeleton protons + N( $\left.\left.\mathrm{CH}_{2} \mathrm{CH}_{3}\right)_{2}\right) ; 1.17\left(\mathrm{~s}, 3 \mathrm{H}, 18-\mathrm{CH}_{3}\right) ; 0.88(\mathrm{~d}, 7.1 \mathrm{~Hz}, 21-$ $\left.\mathrm{CH}_{3}\right) ; 0.80\left(\mathrm{~s}, 3 \mathrm{H}, 19-\mathrm{CH}_{3}\right) ; 0.76\left(\mathrm{~d}, 6.5 \mathrm{~Hz}, 3 \mathrm{H}, 27-\mathrm{CH}_{3}\right) .{ }^{13} \mathrm{C} \mathrm{NMR}$

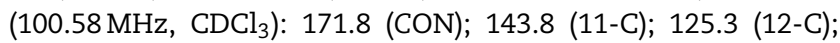
109.5 (22-C); 81.0; 71.3; 67.1; 57.8; 56.9; 53.9; 45.6; 45.1; 42.5; $42.0 ; 38.2 ; 36.3 ; 33.7 ; 31.6 ; 31.5 ; 31.1 ; 30.5 ; 29.3 ; 29.0 ; 21.2 ; 17.3$; 14.2; 13.5; 12.8. MS (m/z): $514(\mathrm{M}+\mathrm{H})^{+}, 496,441,423$, 370. Analysis calculated for $\mathrm{C}_{32} \mathrm{H}_{51} \mathrm{NO}_{4}(\mathrm{M}=513.76)$ : C, 74.81; $\mathrm{H}, 10.01$; $\mathrm{N}, 2.73$. Found: C, 75.02; $\mathrm{H}, 10.23 ; \mathrm{N}, 2.88 . \mathrm{R}_{\mathrm{f}}=0.46\left(\mathrm{CHCl}_{3}\right)$; $R_{\mathrm{f}}=0.55\left(\mathrm{CHCl}_{3} / \mathrm{EtOH}=19 / 1\right) ;$ m.p. $=212-213^{\circ} \mathrm{C}$ (recrystallyzed from ethanol).

2.4.6. 3 3 -Hydroxy-12-N,N-(1',5'-pentadiyl)carboxamido$5 \alpha, 25 R$-spirost-11-ene (10)

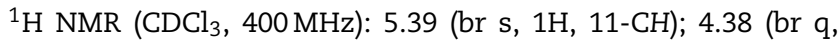
$6.7 \mathrm{~Hz}, 1 \mathrm{H}, 16-\mathrm{CH}) ; 3.20-3.50\left(\mathrm{~m}, 7 \mathrm{H}, 3-\mathrm{CH}, 26-\mathrm{CH}_{2} ; \mathrm{N}\left(\mathrm{CH}_{2}\right)_{2}\right)$; 0.90-2.2 (m, 29H, skeleton protons $\left.+\left(\mathrm{CH}_{2}\right)_{3}\right) ; 0.80(\mathrm{~d}, 6.8 \mathrm{~Hz}, 21-$ $\left.\mathrm{CH}_{3}\right) ; 0.77\left(\mathrm{~s}, 3 \mathrm{H}, 18-\mathrm{CH}_{3}\right) ; 0.72\left(\mathrm{~s}, 3 \mathrm{H}, 19-\mathrm{CH}_{3}\right) ; 0.70(\mathrm{~d}, 6.3 \mathrm{~Hz}$,
3H, 27- $\left.\left.\mathrm{CH}_{3}\right) .{ }^{13} \mathrm{C} \mathrm{NMR} \mathrm{(100.58} \mathrm{MHz,} \mathrm{CDCl}_{3}\right): 170.4$ (CON); 142.9 (11-C); 128.3 (12-C); 109.3 (22-C); 80.7; 70.8; 66.8; 56.7; 53.5; $47.1 ; 46.7 ; 45.2 ; 44.8 ; 41.6 ; 40.5 ; 37.9 ; 36.1 ; 33.4 ; 31.2 ; 31.1$; $30.8 ; 30.1 ; 29.1 ; 28.7 ; 26.5 ; 26.3 ; 25.2 ; 25.0 ; 24.6 ; 20.7 ; 17.2 ; 13.9$; 13.2. MS (m/z): $526(\mathrm{M}+\mathrm{H})^{+}, 508,441,382,344$. Analysis calculated for $\mathrm{C}_{33} \mathrm{H}_{51} \mathrm{NO}_{4}(\mathrm{M}=525.77)$ : C, 75.39; $\mathrm{H}, 9.78 ; \mathrm{N}, 2.66$. Found: C, 75.26; H, 9.59; N, 2.54. $R_{\mathrm{f}}=0.37\left(\mathrm{CHCl}_{3}\right) ; \mathrm{R}_{\mathrm{f}}=0.45$ $\left(\mathrm{CHCl}_{3} / \mathrm{EtOH}=19 / 1\right)$; m.p. $=192-195^{\circ} \mathrm{C}$ (as obtained after column chromatography).

\subsection{7. $3 \beta$-Hydroxy-12-N,N-(1',5'-3-oxapentadiyl)} carboxamido-5 $\alpha, 25 R$-spirost-11-ene (11)

${ }^{1} \mathrm{H} \mathrm{NMR}\left(\mathrm{CDCl}_{3}, 400 \mathrm{MHz}\right): 5.46$ (br s, $\left.1 \mathrm{H}, 11-\mathrm{CH}\right) ; 4.42$ (br q, $6.9 \mathrm{~Hz}, 1 \mathrm{H}, 16-\mathrm{CH}) ; 3.50-3.70\left(\mathrm{~m}, 9 \mathrm{H}, 3-\mathrm{CH}, \mathrm{N}\left(\mathrm{CH}_{2} \mathrm{CH}_{2}\right)_{2} \mathrm{O}\right) ; 3.42$ (br d, $11.0 \mathrm{~Hz}, 1 \mathrm{H}, 26-\mathrm{CH}_{a} \mathrm{H}_{\mathrm{b}}$ ); 3.35 (t, $11.0 \mathrm{~Hz}, 1 \mathrm{H}, 26-\mathrm{CH}_{\mathrm{a}} \mathrm{H}_{\mathrm{b}}$ ); 0.90-2.2 (m, 23H, skeleton protons); 1.20 (s, 3H, 18- $\mathrm{CH}_{3}$ ); 0.92 $\left(\mathrm{d}, 7.1 \mathrm{~Hz}, 21-\mathrm{CH}_{3}\right) ; 0.80\left(\mathrm{~s}, 3 \mathrm{H}, 19-\mathrm{CH}_{3}\right) ; 0.78(\mathrm{~d}, 6.5 \mathrm{~Hz}, 3 \mathrm{H}, 27-$ $\mathrm{CH}_{3}$ ). ${ }^{13} \mathrm{C}$ NMR (100.58 MHz, $\mathrm{CDCl}_{3}$ ): 170.7 (CON); 142.6 (11-C); 126.8 (12-C); 109.6 (22-C); 81.0; 71.3; 67.1; 57.7; 57.1; 53.9; 45.5; $45.1 ; 41.9 ; 38.2 ; 36.4 ; 33.7 ; 31.5 ; 31.1 ; 30.4 ; 29.3 ; 29.0 ; 21.0 ; 17.3$; 14.4; 13.6. MS $(\mathrm{m} / \mathrm{z}): 528(\mathrm{M}+\mathrm{H})^{+}, 510,441,423,384$. Analysis calculated for $\mathrm{C}_{32} \mathrm{H}_{49} \mathrm{NO}_{5}(\mathrm{M}=527.74)$ : $\mathrm{C}, 72.83 ; \mathrm{H}, 9.36 ; \mathrm{N}$, 2.65. Found: C, 72.59; H, 9.50; N, 2.57. $R_{\mathrm{f}}=0.38\left(\mathrm{CHCl}_{3}\right) ; R_{\mathrm{f}}=0.48$ $\left(\mathrm{CHCl}_{3} / \mathrm{EtOH}=19 / 1\right)$; m.p. $=205-206^{\circ} \mathrm{C}$ (as obtained after column chromatography).

\subsubsection{3 -Hydroxy-12-(1'-methoxycarbonyl-ethyl)-} carboxamido- $5 \alpha, 25 R$-spirost-11-ene (12)

${ }^{1} \mathrm{H} \mathrm{NMR}\left(\mathrm{CDCl}_{3}, 400 \mathrm{MHz}\right): 6.13(\mathrm{~d}, 7.7 \mathrm{~Hz}, 1 \mathrm{H}, \mathrm{NH}) ; 5.92(\mathrm{br}$ s, $1 \mathrm{H}, 11-\mathrm{CH}$ ); 4.51 (qi, $7.3 \mathrm{~Hz}, 1 \mathrm{H}, \mathrm{CHCOO}$ ); 4.40 (br q, $7.8 \mathrm{~Hz}$, $1 \mathrm{H}, 16-\mathrm{CH}) ; 3.70\left(\mathrm{~s}, 3 \mathrm{H}, \mathrm{OCH}_{3}\right) ; 3.56(\mathrm{~m}, 1 \mathrm{H}, 3-\mathrm{CH}) ; 3.40$ (br d, $\left.10.9 \mathrm{~Hz}, 1 \mathrm{H}, 26-\mathrm{CH}_{\mathrm{a}} \mathrm{H}_{\mathrm{b}}\right) ; 3.30$ (t, $\left.10.9 \mathrm{~Hz}, 1 \mathrm{H}, 26-\mathrm{CH}_{\mathrm{a}} \mathrm{H}_{\mathrm{b}}\right) ; 0.90-2.2$ (m, 23H, skeleton protons); $1.38\left(\mathrm{~d}, 7.1 \mathrm{~Hz}, 3 \mathrm{H}, \mathrm{CHCH}_{3}\right) ; 1.18$ (s, $\left.3 \mathrm{H}, 18-\mathrm{CH}_{3}\right) ; 0.89\left(\mathrm{~d}, 6.8 \mathrm{~Hz}, 21-\mathrm{CH}_{3}\right) ; 0.73\left(\mathrm{~s}, 3 \mathrm{H}, 19-\mathrm{CH}_{3}\right)$; 0.70 (d, $\left.6.5 \mathrm{~Hz}, 3 \mathrm{H}, 27-\mathrm{CH}_{3}\right) .{ }^{13} \mathrm{C} \mathrm{NMR}\left(100.58 \mathrm{MHz}, \mathrm{CDCl}_{3}\right): 173.8$ (COO); 169.9 (CON); 145.9 (11-C); 128.9 (12-C); 109.2 (22-C); 80.7; 71.0; 66.8; 58.7; 56.9; 53.7; 52.4; 48.7; 47.6; 45.2; 44.8; 42.3; 37.9; 36.1; 33.2; 31.5; 31.2; 30.7; 30.2; 30.0; 29.0; 28.8; 20.0; 18.7; 17.1; 14.2; 13.2. $\mathrm{MS}(\mathrm{m} / \mathrm{z}): 544(\mathrm{M}+\mathrm{H})^{+} ; 526,441,400$, 390. Analysis calculated for $\mathrm{C}_{32} \mathrm{H}_{49} \mathrm{NO}_{6}(\mathrm{M}=543.74)$ : $\mathrm{C}, 70.69 ; \mathrm{H}, 9.08 ; \mathrm{N}$, 2.58. Found: C, 70.51; H, 9.30; N, 2.37. $R_{\mathrm{f}}=0.43\left(\mathrm{CHCl}_{3}\right) ; R_{\mathrm{f}}=0.48$ $\left(\mathrm{CHCl}_{3} / \mathrm{EtOH}=19 / 1\right)$; m.p. $=207-209^{\circ} \mathrm{C}$ (as obtained after column chromatography).

\subsubsection{3 -Hydroxy-12-(1'-methoxycarbonyl-1', $4^{\prime}$ -}

butadiyl)-carboxamido-5 $\alpha, 25 R$-spirost-11-ene (13)

${ }^{1} \mathrm{H} \mathrm{NMR}\left(\mathrm{CDCl}_{3}, 400 \mathrm{MHz}\right): 5.68$ (br s, $\left.1 \mathrm{H}, 11-\mathrm{CH}\right) ; 4.40-4.55$ $(\mathrm{m}, 2 \mathrm{H}, 16-\mathrm{CH}+\mathrm{NCH}) ; 3.70\left(\mathrm{~s}, 3 \mathrm{H}, \mathrm{OCH}_{3}\right) ; 3.50-3.65(\mathrm{~m}, 3 \mathrm{H}, 3-$ $\left.\mathrm{CH}+\mathrm{NCH}_{2}\right) ; 3.42\left(\right.$ br d, $\left.10.7 \mathrm{~Hz}, 1 \mathrm{H}, 26-\mathrm{CH}_{\mathrm{a}} \mathrm{H}_{\mathrm{b}}\right) ; 3.35(\mathrm{t}, 10.7 \mathrm{~Hz}$, $\left.1 \mathrm{H}, 26-\mathrm{CH}_{\mathrm{a}} \mathrm{H}_{b}\right) ; 0.90-2.2\left(\mathrm{~m}, 27 \mathrm{H}\right.$, skeleton protons $\left.+\left(\mathrm{CH}_{2}\right)_{2}\right)$; $1.20\left(\mathrm{~s}, 3 \mathrm{H}, 18-\mathrm{CH}_{3}\right) ; 0.82\left(\mathrm{~d}, 6.8 \mathrm{~Hz}, 21-\mathrm{CH}_{3}\right) ; 0.78(\mathrm{~s}, 3 \mathrm{H}, 19-$ $\left.\mathrm{CH}_{3}\right) ; 0.76\left(\mathrm{~d}, 6.3 \mathrm{~Hz}, 3 \mathrm{H}, 27-\mathrm{CH}_{3}\right) .{ }^{13} \mathrm{C} \mathrm{NMR}\left(100.58 \mathrm{MHz}, \mathrm{CDCl}_{3}\right)$ : 170.2 (CON); 143.8 (11-C); 127.1 (12-C); 109.3 (22-C); 80.7; 71.1; $66.8 ; 58.5 ; 58.1 ; 57.8 ; 56.9 ; 53.9 ; 52.3 ; 52.2 ; 49.6 ; 47.2 ; 45.4 ; 44.8$; $41.5 ; 37.9 ; 36.2 ; 36.0 ; 33.3 ; 31.3 ; 31.2 ; 30.8 ; 30.2 ; 30.1 ; 29.4 ; 29.2$; 29.0; $28.7 ; 25.3 ; 24.5 ; 20.3 ; 17.1 ; 14.2 ; 13.3 . \mathrm{MS}(\mathrm{m} / \mathrm{z}): 570(\mathrm{M}+\mathrm{H})^{+}$, $552,441,423$. Analysis calculated for $\mathrm{C}_{33} \mathrm{H}_{51} \mathrm{NO}_{5}(\mathrm{M}=569.78): \mathrm{C}$, 71.67; H, 9.02; N, 2.46. Found: C, 71.78; H, 9.19; N, 2.30. $R_{\mathrm{f}}=0.36$ $\left(\mathrm{CHCl}_{3}\right) ; R_{\mathrm{f}}=0.40\left(\mathrm{CHCl}_{3} / \mathrm{EtOH}=19 / 1\right) ;$ m.p. $=215-218^{\circ} \mathrm{C}$ (recrys tallyzed from ethanol). 


\section{Results and discussion}

As a part of our ongoing interest in the homogeneous catalytic functionalization of steroids, the introduction of a functional group into position-12 was carried out. The 12-iodo-11-ene derivative, obtained by the transformation of the 12-ketofunctionality of hecogenin (1) via its hydrazone (2), was chosen as model compound for carbonylation reactions (Fig. 1). The 12-iodo-11-ene derivative (3 $\beta$-hydroxy-12-iodo-5 $\alpha .25 \mathrm{R}$-spirost-11-ene, 3) was reacted with carbon monoxide and various primary and secondary amines as $\mathrm{N}$-nucleophiles (tert-butylamine, aniline, diethylamine, pyperidine, morpholine, methyl alaninate or methyl prolinate) or O-nucleophiles (methanol or ethanol) in DMF in the presence of palladiumphosphine 'in situ' catalysts. (The 'in situ' formation of highly active coordinatively unsaturated $\mathrm{Pd}(0)$ catalysts with monoand bidentate phosphines has been published before [14].) The corresponding 12-carboxamido-11-ene derivatives (3 $\beta$ hydroxy-12-N-tert-butyl-carboxamido-5 $\alpha$.25R-spirost-11-ene

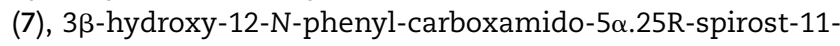
ene (8), 3 $\beta$-hydroxy-12-N,N-diethyl-carboxamido-5 $\alpha .25 \mathrm{R}$-spi-

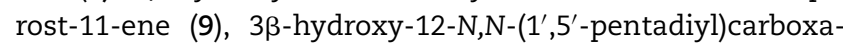

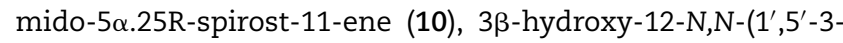
oxapentadiyl)carboxamido-5 $\alpha .25 R$-spirost-11-ene (11), 3 $\beta$-hydroxy-12-N-(1'-methoxycarbonyl-ethyl)-carboxamido- $5 \alpha$. 25R-spirost-11-ene (12), 3ß-hydroxy-12-N,N-(1"'-methoxycarbonyl-1',4'-butadiyl)-carboxamido-5 $\alpha .25 \mathrm{R}$-spirost-11-ene (13)) were synthesized in moderate to good yields depending on
Table 1 - Yields of the aminocarbonylation of 3

Product

Yield $^{\mathrm{a}}$ (isolated yield ${ }^{\mathrm{b}}$ ), \%

\begin{tabular}{rc}
\hline 7 & $>99(92)$ \\
8 & $55(36)$ \\
9 & $95(88)$ \\
10 & $96(64)$ \\
11 & $93(78)$ \\
12 & $>99(90)$ \\
13 & $92(60)$
\end{tabular}

${ }^{a}$ Yields determined by ${ }^{1} \mathrm{H}$ NMR on the reaction mixture.

b Yields obtained after chromatography.

the structure of the amine (Table 1). The lowest yield was obtained with the less basic aniline (35\%), while the highest with tert-butyl-amine and methyl alaninate (92 and $90 \%$, respectively). The application of secondary amines (piperidine and methyl prolinate) resulted in slightly lower yields.

The formation of the carboxamides can be explained by the following reaction mechanism (Fig. 2). The palladium-alkenyl intermediate, which is formed in the oxidative addition of the 'iodo-vinyl' substrate onto the "in situ" formed palladium(0) species, insert carbon monoxide resulting in a palladium-acylcomplex. The highly reactive acyl intermediate undergoes aminolysis with the primary or secondary amine yielding the corresponding carboxamide in the product-forming step.

Using alcohols (methanol, ethanol) as nucleophiles instead of the amines, ester functionality can be introduced into the

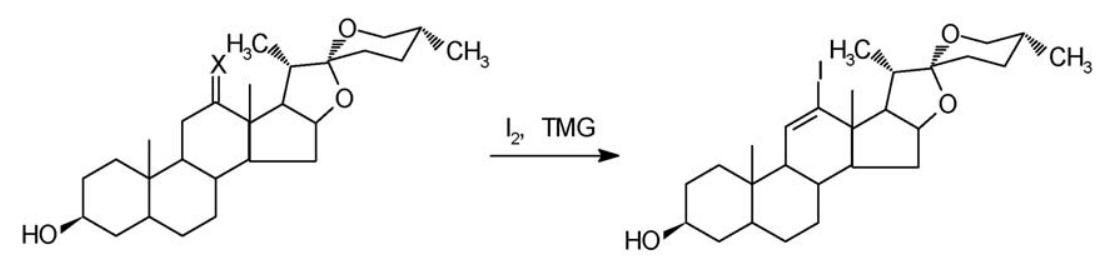<smiles>[X]C(=O)N[C@@H](N)CC</smiles>

3<smiles>[R]OC(C)CCC([R20])N</smiles>

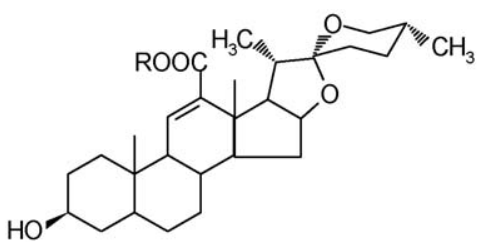

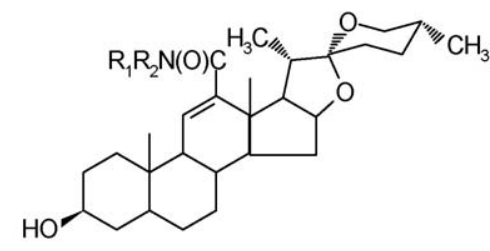

$$
\begin{array}{ll} 
& \mathrm{R} \\
4 & \mathrm{H} \\
5 & \mathrm{CH}_{3} \\
6 & \mathrm{C}_{2} \mathrm{H}_{5}
\end{array}
$$$$
4 \mathrm{H}
$$

Fig. 1 - Synthesis of 12-substituted spirostanes in carbonylation reactions of 12-iodo-11-ene derivative (2). 


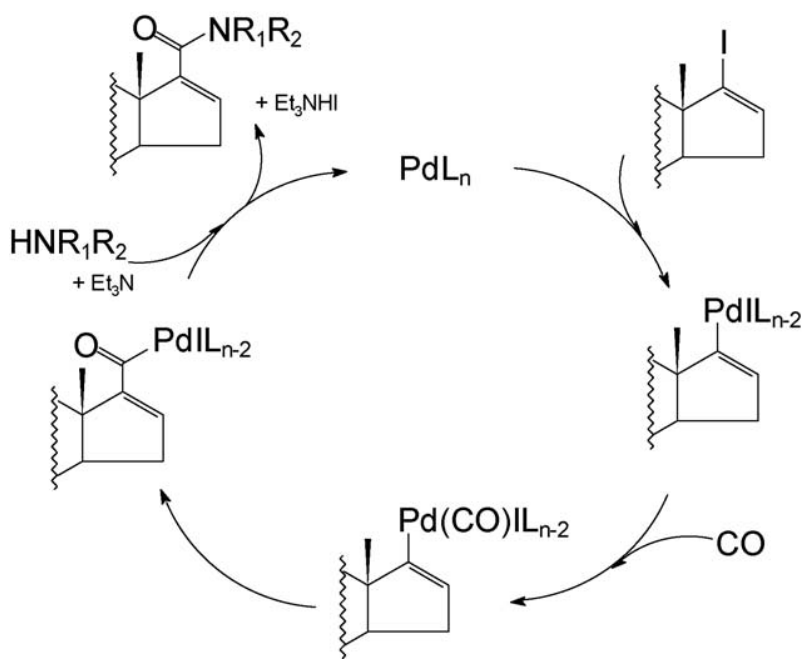

Fig. 2 - A simplified mechanistic representation of the catalytic steps of aminocarbonylation.

steroidal skeleton. Carrying out the alkoxycarbonylation reaction with 3 under similar conditions as the aminocarbonylation reaction, low conversions towards esters (12\% and $10 \%$ for methyl and ethyl ester, respectively) have been obtained and their isolation as pure substance for full characterization failed. (The methyl and ethyl esters ( 5 and 6 , respectively) have been detected by HPLC-MS only.)

Surprisingly, in the presence of alcohols 12-carboxylic acid derivative (4) has been obtained and isolated in yields up to $60 \%$ depending on the reaction conditions. (It has to be noted that carboxylic acids can be synthesized not only via the corresponding esters by hydrolysis but also in direct hydroxycarbonylation [10,15-17].) Similar steroidal acid (17carboxy- $5 \alpha$-androstane) formation was already observed as an unexpected side-reaction with enol-sulfonates [18] and iodoalkenes [19]. In the latter case it was clarified that the corresponding carboxylic acids were produced via the primary formation of carboxylic anhydrides under carbonylation conditions in the presence of the water impurity of the solvent.

As a summary it can be stated, that under appropriate reaction conditions conjugated unsaturated steroidal 12carboxamides can be synthesized in yields of practical interest in palladium catalyzed carbonylation reaction of easily available iodoalkenes as substrates. The strength of the homogeneous carbonylation reaction was shown by the fact that even one of the hindered positions (position 12) can be functionalized in moderate to good yields without any side reactions of the further functionalities.

\section{Acknowledgements}

LK thanks the Hungarian National Science Foundation (OTKA T044800) for the financial support. This publication was also supported by the Hungarian Research and Technology Fund, as well as by the 'Instituto de Cooperacao Cientifica e Technológica' in the framework of the Hungarian-Portuguese Intergovernmental Cooperation (P-5/03).
REFERENCES

[1] Cornils B, Herrmann WA, editors. Applied homogeneous catalysis with organometallic compounds. Weinheim: Wiley-VCH; 1996.

[2] Beller M, Bolm C, editors. Transition metals for organic synthesis, vols. I-II. Weinheim: Wiley-VCH; 1998.

[3] Omae I. Applications of organometallic compounds. New York: Wiley; 1998.

[4] Skoda-Földes R, Kollár L. Transition metal catalyzed reactions in steroid synthesis. Chem Rev 2003;103:4095-129 [and references cited therein].

[5] Holt DA, Levy MA, Metcalf BW, inventors, Smithkline Beecham Co., EP 0,343,954 A2 (1989) (Chem Abstr 1990;112,198890n).

[6] Cacchi S, Ciattini PG, Morera E, Ortar G. Palladium-catalyzed carbonylation of aryl triflates. Synthesis of arenecarboxylic acid derivatives from phenols. Tetrahedron Lett 1986;27:3931-4.

[7] Dolle RE, Schmidt SJ, Kruse LI. Palladium-catalyzed alkoxycarbonylation of phenols to benzoate esters. Chem Commun 1987:904-5.

[8] Holt DA, Levy MA, Ladd DL, Oh H, Erb JM, Heaslip JI, et al. Steroidal A ring aryl carboxylic acids: a new class of steroid 5 $\alpha$-reductase inhibitors. J Med Chem 1990;33:937-42.

[9] Tian W, Lei Z, Chen L, Huang Y. Some new reactions of poly(per)fluoroalkanesulfonyl fluorides with steroidal molecules. J Fluorine Chem 2000;101:305-8.

[10] McGuire MA, Sorenson E, Klein DN, Baine NH. Palladium and nickel catalyzed hydroxycarbonylation of a steroidal bromodiene in the synthesis of episteride, a potent 5 $\alpha$-reductase inhibitor. Synth Commun 1998;28:1611-5.

[11] Petz A, Gálik G, Horváth J, Tuba Z, Berente Z, Pintér Z, et al. Facile, high-yielding synthesis of steroidal crown ethers via palladium-catalyzed carbonylation reaction. Synth Commun 2001;31:335-41.

[12] Barton DHR, O'Brien RE, Sternhell S. A new reaction of hydrazone's. J Chem Soc 1962:470-6.

[13] Barton DHR, Bashiardes B, Fourrey JL. An improved preparation of vinyl iodides. Tetrahedron Lett 1983;24:1605-8.

[14] Csákai Z, Skoda-Földes R, Kollár L. NMR investigation of $\mathrm{Pd}(\mathrm{II})-\mathrm{Pd}(0)$ reduction in the presence of mono- and ditertiary phosphines. Inorg Chim Acta 1999;286:93-7 [and references cited therein].

[15] Cacchi S, Morera E, Ortar G. Palladium-catalyzed carbonylation of enol-triflates. A novel method for one-carbon homologation of ketones to $\alpha, \beta$-unsaturated carboxylic acid derivatives. Tetrahedron Lett 1985;26:1109-12.

[16] Yu MS, Baine NH. Synthesis of 3-carboxy-20-keto-steroid, SB 209963, a 5 $\alpha$-reductase inhibitor, by palladium-catalyzed hydroxycarbonylation. Tetrahedron Lett 1999;40: 3123-4.

[17] Cacchi S, Lupi A. Palladium-catalyzed hydroxycarbonylation of vinyl and aryl triflates: synthesis of $\alpha, \beta$-unsaturated and aromatic carboxylic acids. Tetrahedron Lett 1992;33: 3939-42.

[18] Zhu Z, Tian W, Liao Q. A practical procedure for chemo- and regioselective conversion of steroid 3-ketones into the corresponding enol sulfonates using 3-oxa-octafluoropentanosulfonyl fluoride. Tetrahedron Lett 1996;37: 8553-6.

[19] Skoda-Földes R, Csákai Z, Kollár L, Szalontai G, Horváth J, Tuba Z. Homogeneous catalytic dehalodimerization of 17-iodo- $\Delta^{16}$-ene steroids. Steroids 1995;60: 786-90. 